\title{
[Industrial paper] Deployment process for Modelica-based models
}

\author{
Takashi Iwagaya $^{1}$ Chad Schmitke $^{2}$ Tetsu Yamaguchi ${ }^{2}$ \\ ${ }^{1}$ Cybernet Systems Co., Ltd., Japan, i wagaya@ cybernet.co.jp \\ ${ }^{2}$ Maplesoft, Canada, \{cschmitke, tetsuy\} @maplesoft.com
}

\begin{abstract}
This paper introduces Maplesoft's solutions for deploying Modelica-based models for non-experts of simulation. In order to apply models to analysis, either non-experts need to learn how to use simulation tools, or simulation experts need to prepare easy-of-use GUIs, like Excel and Web technologies. Maplesoft's solutions, MapleSim Explorer and MapleSim Server, allow more flexible and rapidly developed analysis tools for non-experts. These solutions encourage wide use of simulation models.
\end{abstract}

Keywords: Model execution tool, Deployment, Webbase, Cloud

\section{Introduction}

Modelica-based models should be applied to analyze design, and explore the design space of parameters and determine design parameters. However, it is often difficult to use the simulation tools for general design engineers who do not have simulation expertise. One solution for this problem is to educate engineers, but they don't have enough time to learn new tools and methodologies. To solve this difficulty, simulation experts can develop models and ease-of-use GUI applications in many cases (Figure 1). Thus, it takes time to provide analysis environment to non-expert users.
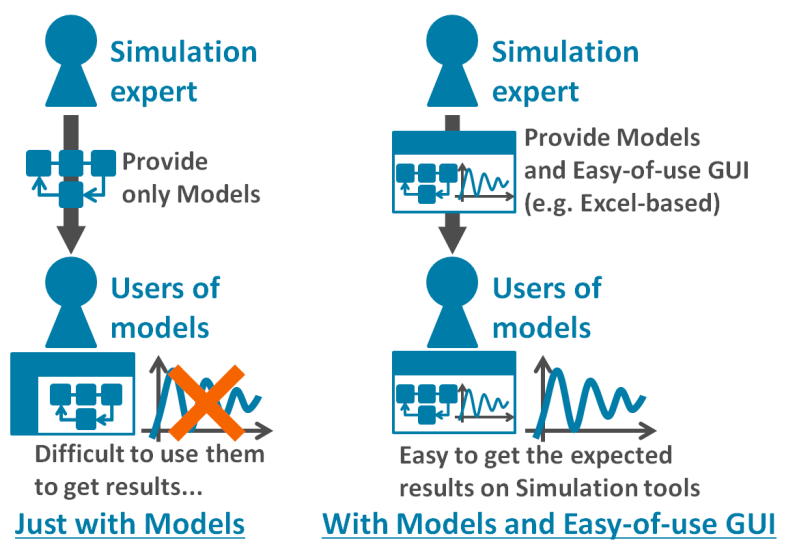

Figure 1. How to provide models to the non-experts of simulation.

The other difficulty for non-experts is how to utilize models developed by experts. The usage of them must be related to given design tasks. Non-experts may have difficulties when parameters and evaluation points for the design do not correspond directly with the parameters and variables of simulation models. In other words, post processing may be required to evaluate the design. For example, frequency analysis may be applied to simulation results in the time domain. Additionally, parameters of a model tuned by design engineers may be several selected parameters in a model. Thus, the limited accessibility in the parameter setting is sometimes useful for non-experts. In these cases, simulation experts will provide customized analysis tools for each design tasks, which make operations of tools easier for non-experts.

In the following section, we introduce Maplesoft's solutions to simplify such deployment framework, and expand the use of Modelica models.

\section{Deployment types and use-cases}

Maplesoft product can offer three types of deployment approach other than that based on code generation (for example, using FMI) (Figure 2). In the figure, models are Modelica-based models created in MapleSim, and analysis tools mean pre and post processing tools which are connected to models.

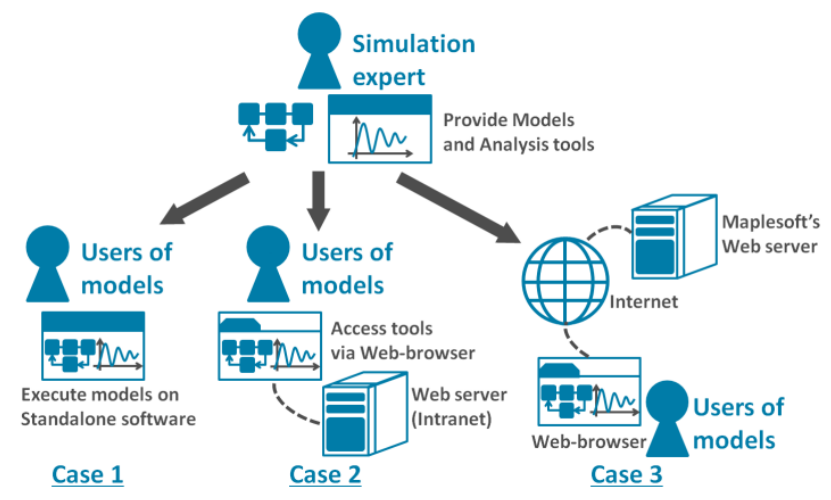

Figure 2. Three types of deployment with Maplesoft products.

The three types of deployment are :

- Case 1 : With Standalone software, MapleSim Explorer (MapleSim Explorer, 2018). Users need to install software and store models and analysis tools developed by experts on their own computer 
- Case 2 : Web-based environment, MapleSim Server (MapleSim Server, 2018). Software, models and analysis tools are installed on Web server. User can access the analysis tools via Web-browser.

- Case 3 : Cloud-based environment, MapleCloud (MapleCloud, 2018). Analysis tools containing models are uploaded to cloud platform. Users can execute tools on cloud and download them to their own environment.

As fundamental capability of Maplesoft product suite, it is easy to build user-defined analysis tools in Maple, which consist of calculation procedures and GUI, to set parameters to defined Modelica-based models and apply post processing with the simulation results. Additionally, one of Maple's file formats (.maple file) can contain Modelica-based models (MapleSim model file .msim and Modelica code .mo). And, MapleSim model file (.msim) also can store analysis tools created in Maple. Thus, a packed file consisted of model and analysis tool can be easily uploaded to MapleCloud or Web-server, and shared between simulation experts and non-expert users. Thus, users do not need to worry about connectivity between models and analysis tools.

Advantages of above three types of deployment approach are outlined in the following sections.

\subsection{Case 1 : MapleSim Explorer}

MapleSim Explorer gives almost same usability as MapleSim itself (Figure 3). Users can set value of parameters, simulate models and use analysis tools, but cannot modify model structure and save any changes for value of parameters in MapleSim Explorer.

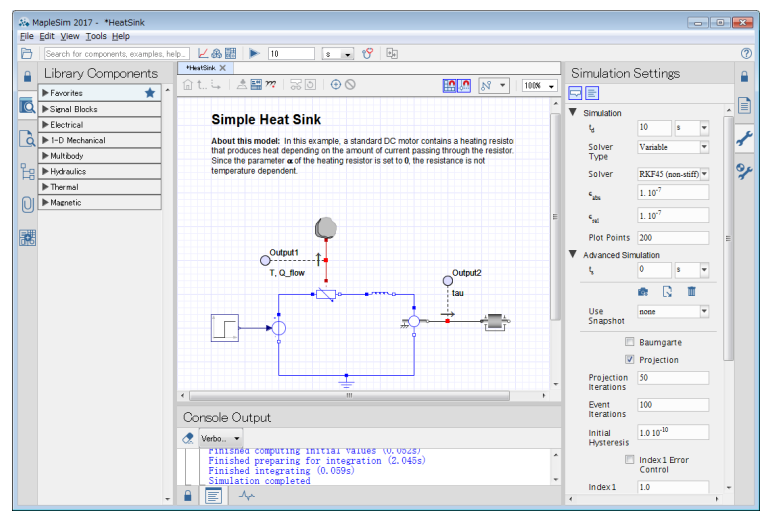

Figure 3. GUI of MapleSim Explorer

The benefits of using MapleSim Explorer are :

- There is no risk of errors for inadvertent user changes models or analysis tools.

- Especially in terms of using models and analysis tools, user experience in MapleSim Explorer is almost same as MapleSim. Therefore, simulation experts can easily teach non-experts on how to use given application.
On the other hand, this workflow suffers from some minor development inconveniences. Users need to install software in local computer. Additionally, version management of models and analysis tools should be handled by simulation experts or tool manager. In other words, these are weaknesses compared with two other deployment approaches.

\subsection{Case 2 and 3 : MapleSim Server and MapleCloud}

MapleSim Server and MapleCloud are built on top of the same underlying technology. User experiences about execution for non-experts are the same in both case. Users can use GUI components on analysis tools developed in Maple (Figure 4).

For users in either environments, they do not need to install anything locally; they simply access the specified URL in their web browser. Major difference between two environments is whether person who manages the analysis tools also needs to manage the web server or not.

As other benefit of MapleSim Server, it can be integrated with existing web-based systems in user's organization especially about GUI because of Web technologies.

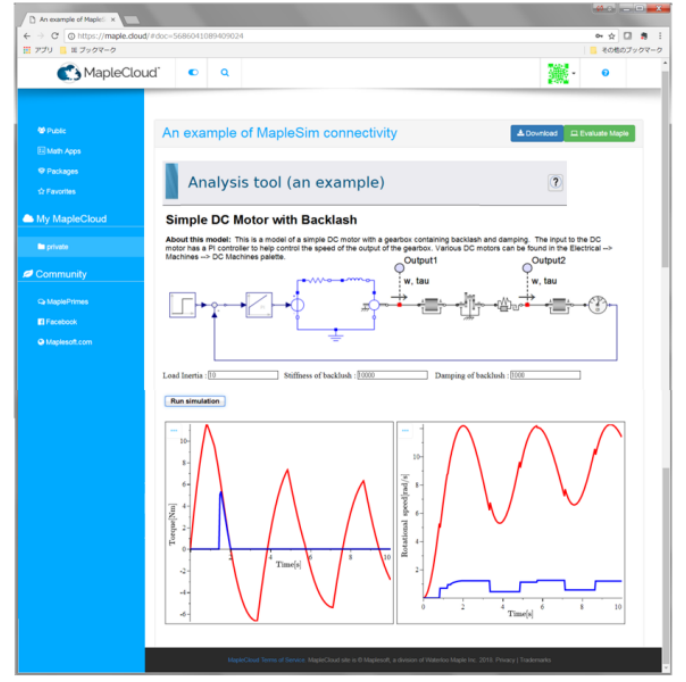

Figure 4. An example of analysis tool on MapleCloud

\section{Workflow of Analysis tools development}

In this section, typical workflow of Analysis tools development is shown by using a simple case study. simulation experts need to develop models on MapleSim and analysis tools on Maple for all types of deployment (Figure 5). First, models are created with components which are defined by Modelica as general Modelica based modeling tool. Next step is to develop analysis tools in Maple. GUI in tools is easily developed by using Maple's predefined GUI components. And, to define the behavior of each components, action code can be implemented in the compoment. 


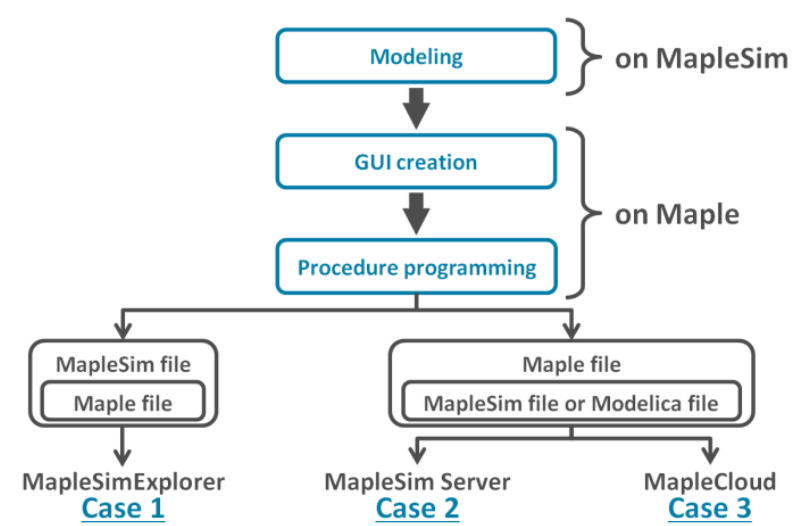

Figure 5. Workflow of Analysis tools development

Especially, connection between model and analysis tool is established by using three options:

1. Attach one or multiple Maple files of analysis tool (.mw or .maple) to MapleSim model file (.msim). Then use the MapleSim component of Maple's GUI component to establish connection between model and analysis tools.

2. Attach one or multiple MapleSim model files (.msim or .mo) to Maple file of analysis tool (.maple). And then use Maple command in Maple file to establish connection between models and analysis tools. Figure 6 shows this case.

3. Use Maple command in Maple file of analysis tool (.mw or .maple) to specify file stored path of MapleSim model file (.msim or .mo), which is located at outside the Maple file.

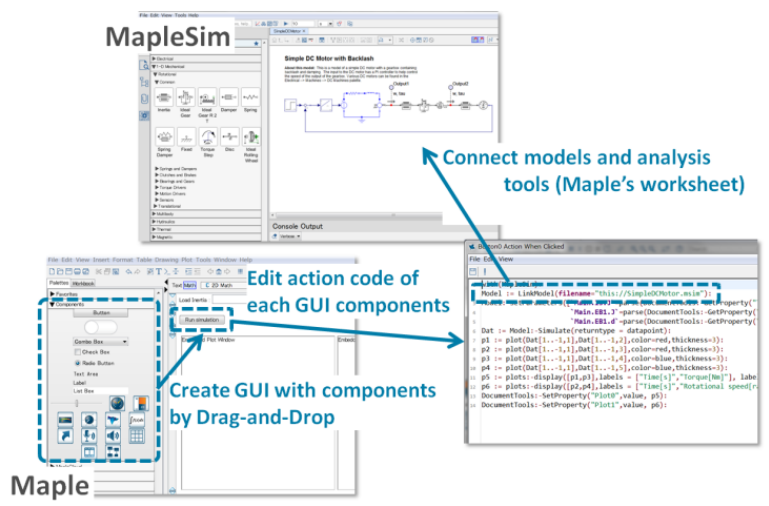

Figure 6. Modeling and Analysis tools development

First option in above case is for using MapleSim Explorer, which is Case 1 in Figure 2 and 5. As typical workflow, simulation experts can simply share the model file which contains one or multiple analysis tools with non-expert users. Then, they can get several analysis results instantly for given target design, which can be expressed within the model. (Figure 7).

Second option can be used for MapleSim Server and MapleCloud, which are corresponding to Case 2 and Case 3. Simulation experts need to upload analysis tool that contains one or multiple models to own web server or MapleCloud's. Then, non-expert users just need to

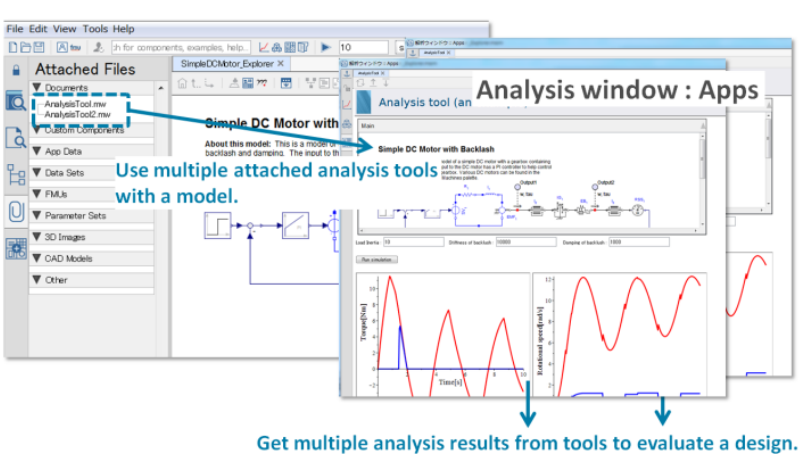

Figure 7. Using MapleSim Explorer

access the specified URL from their web browsers to run analysis tools. With this framework, it is possible to analyze multiple attached models and to do multiple types of analysis which is implemented in the associated Maple file.

Third option is effective for simple routine tasks with different models only in Case 2. For example, if the definition of interface is fixed, the code generation (FMU and S-Function) can be applied, though it is not analysis task.

Simulation experts need to select what the appropriate file structure is, based on use-cases when they develop models and analysis tools. Meantime, models and analysis tools can be used in common use case for all types of deployment, hence simulation experts can provide analysis environment which is suitable for non-expert users' demand appropriately.

\section{Conclusions}

In this paper, we introduced typical deployment approaches by Maplesoft product suite. One of difficulties that may occur when simulation experts provide models and analysis tools to non-experts, can be clarified with described approaches. The key factor of how simulation experts can develop analysis tools efficiently is related to flexible file structure for connection between model files and analysis tool files. In this context, Maplesoft technologies allow to access Maple files directly from web browser. We are already working closely with some customers in Japan to see practical increase of usage for Modelica-based models by providing these deployment approaches discussed in this paper.

\section{References}

\author{
MapleCloud (2018): \\ https://maple.cloud \\ MapleSim Explorer (2018): \\ https://www.maplesoft.com/products/maplesimexplorer/ \\ MapleSim Server (2018): \\ https://www.maplesoft.com/products/maplesimserver/
}

